\title{
Digitalisierung und Resilienz in der Food Supply Chain
}

\author{
Manfred Hofmeier - Ulrike Lechner
}

Eingegangen: 3. Juni 2020 / Angenommen: 10. August 2020 / Online publiziert: 2. September 2020 (C) Der/die Autor(en) 2020

Zusammenfassung Im durch das Bundesministerium für Bildung und Forschung und das österreichische Bundesministerium für Landwirtschaft, Regionen und Tourismus geförderten Forschungsprojekt NutriSafe wurde die Studie „NutriSafe Monitor - Resilienz und Blockchain-Technologie in Lebensmittelproduktion und -logistik" durchgeführt. Entscheider in Unternehmen der Lebensmittelproduktion und -logistik im deutschsprachigen Raum wurden in einer Online-Umfrage zu den Themen Resilienz, Digitalisierung, Transparenz und Produktrückverfolgung sowie Chancen und Risiken von Blockchain-Technologie befragt. Der vorliegende Beitrag präsentiert die Ergebnisse der Online-Umfrage zur Resilienz und erweitert diese um bivariate Analysen zur Resilienz im Hinblick auf die Digitalisierung. Resilienz wird hier verstanden als Komplex aus Robustheit und Anpassungsfähigkeit und flexible Reaktion auf Störungen und disruptive Veränderungen. Bisher ist wenig über das Thema Resilienz in kleinen und mittleren Unternehmen der Lebensmittelproduktion und -logistik bekannt. Der vorliegende Beitrag füllt diese Lücke und analysiert Faktoren der Resilienz (Unterbrechungen in der Wertschöpfungskette, Störungen im Unternehmen, Verwundbarkeit und Bewältigungskapazitäten), Indikatoren der Resilienz sowie die Zusammenhänge mit dem Digitalisierungsgrad in diesen Unternehmen. Ziel der Analyse ist ein Lagebild der Resilienz und der Digitalisierung in Lebensmittelproduktion und -logistik und eine Grundlage zu schaffen, um mögliche Auswirkungen voranschreitender Digitalisierung auf die Lebensmittelversorgung abschätzen zu können.

Schlüsselwörter Digitalisierung · Resilienz · Wertschöpfungsketten · Lebensmittelproduktion · Logistik

\footnotetext{
M. Hofmeier $(\bowtie) \cdot$ U. Lechner

Institut für Schutz und Zuverlässigkeit, Universität der Bundeswehr München, München, Deutschland

E-Mail: manfred.hofmeier@unibw.de
} 


\title{
Digitization and Resilience in the Food Supply Chain
}

\begin{abstract}
In the NutriSafe research project, funded by the German Federal Ministry of Education and Research and the Austrian Federal Ministry for Agriculture, Regions and Tourism, the study "NutriSafe Monitor-Resilience and Blockchain Technology in Food Production and Logistics" was conducted. Decision-makers from companies in food production and logistics in German-speaking countries were surveyed online on the topics of resilience, digitization, transparency and product traceability as well as the opportunities and risks of blockchain technology. This paper presents the results of the survey on resilience and extends the data analysis by bivariate analyses on the topic of resilience with regard to digitization. Resilience is understood here as a complex of robustness and adaptability or flexible reaction to disruptive events. Until now, little is known about the topic of resilience in small and medium-sized enterprises in food production and logistics. The present paper fills this gap and analyses factors of resilience (supply chain interruptions, disruptions within the company, vulnerability and coping capacities) as well as correlations with the degree of digitization in these companies. The aim of this analysis is to get a picture of resilience and digitization in food production and logistics and to create a basis to estimate possible effects of the proceeding digitization on food supply.
\end{abstract}

Keywords Digitization $\cdot$ Resilience $\cdot$ Supply chains $\cdot$ Food production $\cdot$ Logistics

\section{Einleitung}

Im durch das Bundesministerium für Bildung und Forschung (BMBF) und das österreichische Bundesministerium für Landwirtschaft, Regionen und Tourismus (BMLRT) geförderten Forschungsprojekt NutriSafe (Sicherheit in der Lebensmittelproduktion und -logistik durch die Distributed-Ledger-Technologie) forschen Universitäten, Unternehmen und Behörden daran, die Lebensmittelproduktion und deren Logistik unter Nutzung von Distributed-Ledger-Technologie resilienter zu machen (Reimers et al. 2019; NutriSafe 2019).

In Lebensmittelproduktion und -logistik wirken im Themenkomplex Resilienz sowohl Fragen der Lebensmittelsicherheit als auch der IT-Sicherheit zusammen. So gingen im Jahr 2018 beim europäischen Rapid Alert System for Food and Feed (RASFF) 3699 Meldungen ein, von denen 1118 Meldungen der Stufe ,alert“ entsprachen, wobei über die Jahre hinweg besonders bei dieser Meldestufe ein starker Anstieg zu verzeichnen ist (Europäische Kommission 2019). In der Auswertung des Bundesamts für Sicherheit in der Informationstechnik (BSI) (2019) über die Häufigkeit von Cybervorfällen in Kritischen Infrastrukturen für das Jahr 2019 ist der Sektor Ernährung derjenige mit den wenigsten IT-Sicherheitsmeldungen. Das zur Meldung verpflichtende IT-Sicherheitsgesetz (Deutscher Bundestag 2015) betrifft Unternehmen, die mehr als 434.500 Tonnen bzw. mehr als 350 Mio. Liter im Jahr produzieren oder verarbeiten (Bundesministerium des Innern 2016). Im Jahr 2019 waren 35 Organisationen im Sektor Ernährung nachweispflichtig (Bundesamt für Sicherheit in der Informationstechnik 2019). Der Sektor Ernährung ist jedoch 
von kleinen und mittleren Unternehmen (KMU) sowie Verfahren der kontinuierlichen Belieferung geprägt (Platz 2005). Die Konzepte des Projekts NutriSafe sollen die Resilienz in Lebensmittelproduktion und -logistik erhöhen und dabei vor allem KMU ansprechen. Wenig ist über Resilienz und Digitalisierung in diesem Bereich bekannt.

Als Grundlage für diese Arbeit wird die Definition des Resilienzbegriffs verwendet, die wortgleich vom Bundesamt für Bevölkerungsschutz und Katastrophenhilfe (BBK) und dem BSI verwendet wird: ,Resilienz ist die Fähigkeit eines Systems, mit Veränderungen umgehen zu können. Resilienz bedeutet Widerstandsfähigkeit gegen Störungen jeder Art, Anpassungsfähigkeit an neue Bedingungen und eine flexible Reaktion auf Veränderungen mit dem Ziel, das System - z. B. einen Betrieb oder einen Prozess - aufrecht zu erhalten“ (Bundesamt für Bevölkerungsschutz und Katastrophenhilfe 2011; Bundesamt für Sicherheit in der Informationstechnik o.J.). Als Digitalisierung wird in der vorliegenden Arbeit die Unterstützung oder vollständige Umsetzung von Unternehmensprozessen durch Informationssysteme verstanden.

In der im Rahmen von NutriSafe entstandenen Studie „NutriSafe Monitor - Resilienz und Blockchain-Technologie in Lebensmittelproduktion und -logistik“ (NutriSafe 2020) wurden Entscheider in Unternehmen der Lebensmittelproduktion und -logistik zu den Themen Resilienz, Rückverfolgungen und Rückrufe, Transparenz, Digitalisierung sowie Chancen und Risiken von Blockchain-Technologie befragt. Im vorliegenden Beitrag werden die Daten zum Thema Resilienz analysiert, welche im Rahmen der Studie erhoben wurden, und Resilienzindikatoren werden auf Zusammenhänge mit dem Digitalisierungsgrad in Unternehmen untersucht. So kann für die Food Supply Chain ein Lagebild der Resilienz und Digitalisierung erstellt werden und mögliche Auswirkungen voranschreitender Digitalisierung auf die Lebensmittelversorgung können abgeschätzt werden.

\section{Datenbasis}

Für die Monitor-Studie wurden Unternehmen aus Lebensmittelproduktion und -logistik im deutschsprachigen Raum (Deutschland, Österreich, Schweiz) befragt. Der Fragebogen mit 31 Fragen wurde im Frühjahr 2019 unter Einbezug aller Projektpartner des Forschungsprojekts NutriSafe konzipiert. Die Umfrage fand von Juni 2019 bis Oktober 2019 als Onlinefragebogen statt. 108 Unternehmen nahmen an der Umfrage teil, wovon 66 Teilnehmer den Fragebogen vollständig bearbeiteten.

\subsection{Umfrageteilnehmer}

Die Umfrageteilnehmer der Studie sind Entscheider in Unternehmen der Lebensmittelproduktion und -logistik. Mitglieder von Geschäftsführungen und Personen in der Leitung von Produktion, Logistik oder Qualitätssicherung brachten ihre Einschätzungen ein.

Zur Einordnung der teilnehmenden Unternehmen in Branchenbereiche dienen die Kategorien aus der Statistischen Systematik der Wirtschaftszweige in der Europäi- 
Tab. 1 Branchenbereiche und Rollen in der Wertschöpfungskette

\begin{tabular}{|c|c|c|c|}
\hline $\begin{array}{l}\text { Rolle in der } \\
\text { Wertschöpfungskette }\end{array}$ & $\begin{array}{l}\% \text { der Be- } \\
\text { fragten }\end{array}$ & Branchenbereich (NACE Rev. 2) & $\begin{array}{l}\% \text { der Be- } \\
\text { fragten }\end{array}$ \\
\hline \multirow[t]{2}{*}{$\overline{\text { Erzeuger }}$} & 24,1 & Landwirtschaft und Jagd (A 01) & 21,7 \\
\hline & & Fischerei und Aquakultur (A 03) & 6,0 \\
\hline \multirow{10}{*}{$\begin{array}{l}\text { Produktion und } \\
\text { Weiterverarbeitung }\end{array}$} & 68,7 & Schlachten und Fleischverarbeitung (C 10.1) & 16,9 \\
\hline & & Fischverarbeitung (C 10.02) & 12,0 \\
\hline & & Obst- und Gemüseverarbeitung (C 10.03) & 13,3 \\
\hline & & $\begin{array}{l}\text { Herstellung von pflanzlichen und tierischen } \\
\text { Ölen und Fetten (C 10.4) }\end{array}$ & 7,2 \\
\hline & & Milchverarbeitung (C 10.5) & 10,8 \\
\hline & & $\begin{array}{l}\text { Mahl- und Schälmühlen, Herstellung von } \\
\text { Stärke und Stärkeerzeugnissen (C 10.06) }\end{array}$ & 6,0 \\
\hline & & Herstellung von Back- und Teigwaren (C 10.7) & 15,7 \\
\hline & & Herstellung von Getränken (C 11) & 4,8 \\
\hline & & Herstellung v. sonst. Nahrungsmitteln (C 10.8) & 24,1 \\
\hline & & Herstellung von Futtermitteln (C 10.9) & 9,6 \\
\hline \multirow[t]{2}{*}{ Handel } & 27,7 & Großhandel (G 46) & 16,9 \\
\hline & & Einzelhandel (G 47) & 15,7 \\
\hline $\begin{array}{l}\text { Transport und Lo- } \\
\text { gistik }\end{array}$ & 13,3 & Transport und Logistik $(\mathrm{H})$ & 13,3 \\
\hline Gastronomie & 15,7 & Gastronomie (I 56) & 15,7 \\
\hline
\end{tabular}

schen Gemeinschaft (NACE Rev. 2) als Muster (Eurostat 2008). Unternehmen sind dabei häufig gleichzeitig in mehreren Branchenbereichen aktiv.

Für die Vergleiche zwischen verschiedenen Typen von Akteuren in der Wertschöpfungskette wurden die Kategorien in größere Gruppen nach der Rolle in der Wertschöpfungskette zusammengefasst (Tab. 1). Der mehrheitliche Anteil der Befragten ist in der Produktion bzw. Weiterverarbeitung von Lebensmitteln tätig (68,7\%). 27,7\% sind im Handel und 24,1\% sind als Erzeuger tätig. Im Bereich Transport und Logistik betätigen sich $13,3 \%$ der befragten Unternehmen und im Bereich Gastronomie 15,7\%.

Laut EU-Empfehlung der Kommission 2003/361 sind kleine und mittlere Unternehmen (KMU) Unternehmen, ,die weniger als 250 Personen beschäftigen und die entweder einen Jahresumsatz von höchstens 50 Mio. EUR erzielen oder deren Jahresbilanzsumme sich auf höchstens 43 Mio. EUR beläuft" (Europäische Kommission 2003). Nach dieser Definition sind 66,7\% der befragten Unternehmen KMU.

Bei den meisten befragten Unternehmen sind die meisten Bereiche bzw. Prozesse mindestens digital unterstützt, bei mehr als $20 \%$ der Unternehmen sind sie sogar vollkommen digitalisiert (Abb. 1). Dabei besteht kein nennenswerter Unterschied zwischen KMU und größeren Unternehmen. Am meisten fortgeschritten ist die Digitalisierung beim Zahlungsverkehr. Da die Umfrageteilnehmer zu großen Teilen digital angesprochen wurden (z. B. Social Media, Email) und potentiellen Teilnehmern vorab ein digitaler Themenbezug kommuniziert wurde, können die Ergebnisse der Studie durch Selbstselektion zugunsten der Digitalisierung verzerrt sein und nicht ohne weiteres auf die Grundgesamtheit übertragen werden. 
Wareneingangsdokumentation

Warenausgangsdokumentation

Dokumentation der Qualitätssicherung

Zahlungsverkehr

Betriebliche Ressourcensteuerung (Material und Personal)

Auftrags- und Vertragsmanagement

Produktionssteuerung

Kommunikation mit Geschäftspartnern

Informationsquellen

(z.B. Gefahrstoffe, Allergene)

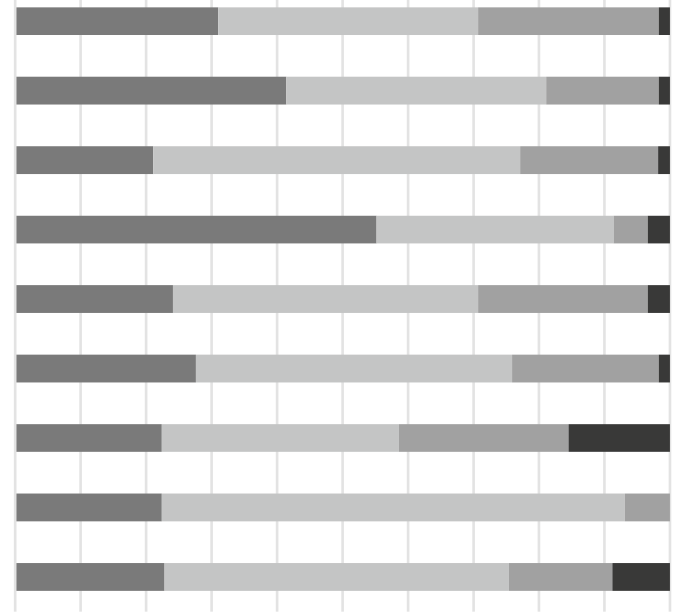

vollkommen digital teils digital $\quad$ nicht digital $\quad$ keine Angabe

Abb. 1 Digitalisierung der Bereiche/Prozesse in den Unternehmen (NutriSafe 2020)

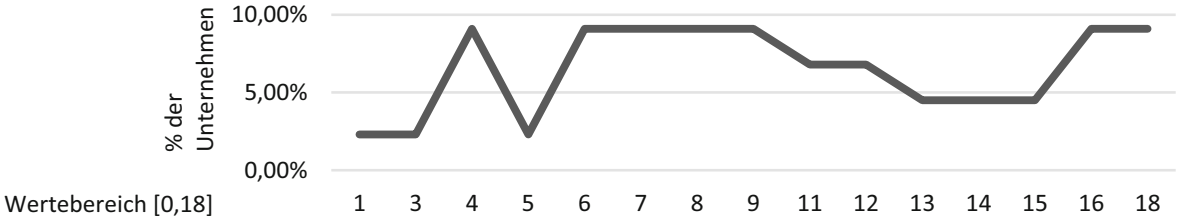

Abb. 2 Verteilung der Umfrageteilnehmer über den Wertebereich des Digitalisierungsgrad-Index

Um in der bivariaten Analyse Zusammenhänge zwischen dem Digitalisierungsgrad und Resilienzindikatoren untersuchen zu können, wurde ein Index gebildet, welcher den Digitalisierungsgrad als Zahl abbildet. Hierfür wurden die Werte für die Digitalisierung der Unternehmensbereiche mit Zahlenwerten versehen (nicht digital $=0$, teils digital $=1$, vollkommen digital $=2$ ) und über alle Unternehmensbereiche addiert, so dass ein einzelner Zahlenwert den Digitalisierungsgrad des jeweiligen Unternehmens im Wertebereich [0,18] angibt (für die Verteilung über den Wertebereich siehe Abb. 2).

Der Digitalisierungsgrad unterscheidet sich nach der Rolle in der Wertschöpfungskette. So liegt der Mittelwert des Digitalisierungsgrad-Index bei Erzeugern bei 7,54, bei Produzenten oder Weiterverarbeitern bei 9,69, beim Handel bei 8,87, bei Transport und Logistik bei 7,50 und in der Gastronomie bei 6,75. 


\section{Resilienz}

In Wertschöpfungsketten gibt es unterschiedliche Konzepte der Resilienz auf unterschiedlichen Ebenen (Ponis und Koronis 2012; Christopher und Peck 2004). So kann Resilienz in Wertschöpfungsketten von Lebensmitteln unter anderem auf folgenden Ebenen betrachtet werden: Resilienz der Versorgung der Bevölkerung mit Lebensmitteln als Ganzes, Resilienz einer bestimmten Wertschöpfungskette für ein spezifisches Lebensmittel, Resilienz eines in die Wertschöpfungskette eingebetteten Betriebes. In Bezug auf die Resilienz eines Betriebes, welche für die vorliegende Arbeit maßgeblich ist, lässt sich das Konstrukt Resilienz untergliedern in:

- Resilienz in Bezug auf Unterbrechungen in der Lieferkette (upstream und downstream)

- Resilienz in Bezug auf Störungen innerhalb des Unternehmens.

Für die Analyse wird Resilienz im NutriSafe Monitor zudem als Konstrukt aus Verwundbarkeit und Bewältigungskapazitäten operationalisiert.

\subsection{Unterbrechungen in der Wertschöpfungskette}

Unterbrechungen in der vor- und nachgelagerten Lieferkette treten bei den meisten befragten Unternehmen auf, jedoch geben die meisten Unternehmen an, dass Unterbrechungen selten oder $a b$ und zu vorkommen (Tab. 2). Nur ein kleiner Teil der Befragten (3,3\% für die vorgelagerte Kette, 1,7\% für die nachgelagerte Kette) gibt Unterbrechungen als häufig an.

Zwischen dem Digitalisierungsgrad und Unterbrechungen in der vor- und nachgelagerten Lieferkette besteht ein schwacher positiver Zusammenhang (Tab. 2). Bei der vorgelagerten Lieferkette ist der Zusammenhang signifikant, bei der nachgelagerten Kette ist der Zusammenhang nicht weit vom Signifikanzbereich entfernt. Bezieht man bei der Analyse des Zusammenhangs der Digitalisierung mit Unterbrechungen in der vorgelagerten Kette die Rolle der Unternehmen in der Wertschöpfungskette mit ein, bleibt dieser Zusammenhang bei Erzeugern (Kendall's Tau b=0,357, Signifikanz $=0,066$ ) und Transport/Logsitik (Kendall's Tau b=0,816, Signifikanz =0,061) bestehen, jedoch nicht statistisch signifikant. Innerhalb der anderen Branchenberei-

Tab. 2 Unterbrechungen in der Lieferkette

\begin{tabular}{|c|c|c|c|c|c|c|c|}
\hline & \multicolumn{5}{|c|}{$\begin{array}{l}\text { Häufigkeit von Unterbrechungen in der } \\
\text { Lieferkette }\end{array}$} & \multicolumn{2}{|c|}{$\begin{array}{l}\text { Einfluss des Digitalisie- } \\
\text { rungsgrades (Korrelation) }\end{array}$} \\
\hline & $\begin{array}{l}\text { Gar } \\
\text { nicht }\end{array}$ & Selten & $\begin{array}{l}\mathrm{Ab} \\
\text { und } \\
\mathrm{zu}\end{array}$ & Häufig & $\begin{array}{l}\text { Weiß } \\
\text { nicht }\end{array}$ & $\begin{array}{l}\text { Kendall's } \\
\text { Tau b }\end{array}$ & Signifikanz \\
\hline $\begin{array}{l}\text { Unterbrechungen in } \\
\text { der vorgelagerten Lie- } \\
\text { ferkette (Zulieferer) }\end{array}$ & $21,70 \%$ & $35,00 \%$ & $33,30 \%$ & $3,30 \%$ & $6,70 \%$ & 0,205 & 0,049 \\
\hline $\begin{array}{l}\text { Unterbrechungen in } \\
\text { der nachgelagerten } \\
\text { Lieferkette (Abneh- } \\
\text { mer) }\end{array}$ & $23,30 \%$ & $36,70 \%$ & $28,30 \%$ & $1,70 \%$ & $10,00 \%$ & 0,194 & 0,063 \\
\hline
\end{tabular}


che besteht kein Zusammenhang zwischen dem Digitalisierungsgrad und Unterbrechungen in der vorgelagerten Kette. Es ist denkbar, dass kein kausaler Zusammenhang zwischen den beiden Eigenschaften besteht, sondern vielmehr die Art und Ausprägung des jeweiligen Unternehmens ausschlaggebend für beide Variablen ist.

\subsection{Ursachen für Störungen im Unternehmen}

Die häufigsten Ursachen für Störungen im Unternehmen sind menschliche Fehler, Missverständnisse mit Partnern, organisatorische Prozessfehler und Qualitätsprobleme bei Eingangswaren (Abb. 3). Etwa ein Drittel der befragten Unternehmen war schon einmal von Naturkatastrophen betroffen und physische Störungen, wie beispielsweise mechanische Defekte oder blockierte Zufahrten, spielen bei etwa zwei Dritteln eine Rolle. Systemfehler und -ausfälle kommen bei 13,6\% ab und zu und bei $54,2 \%$ selten vor. Sabotage und Cyberangriffe sind insgesamt selten, kommen aber vor, womit sich nach diesen Daten für den Ernährungssektor eine günstigere Bedrohungslage abzeichnet als in der Studie ,Monitor 2.0 IT-Sicherheit Kritischer Infrastrukturen“ (VeSiKi 2018), die Kritische Infrastrukturen sektorenübergreifend betrachtet. Eine mögliche Ursache dafür sind die kleinen Unternehmensgrößen und die vergleichsweise geringe Digitalisierung im Ernährungssektor.

Betrachtet man den Zusammenhang zwischen Digitalisierungsgrad und der Häufigkeit von Störungen (Tab. 3), fällt auf, dass ein schwach positiver Zusammenhang mit geringer Signifikanz bei Systemfehlern und -ausfällen besteht. In Bezug auf die Häufigkeit von Cyberangriffen besteht kein Zusammenhang. Ein negativer Zusammenhang besteht vor allem bei Missverständnissen mit Partnern und fehlender bzw. fehlerhafter Dokumentation, wobei diese Zusammenhänge schwach ausgeprägt sind und knapp nicht im Signifikanzbereich liegen. Auffällig ist, dass die Zusammenhangsmaße bei technischen Ursachen positiv und bei menschlichen bzw. organisatorischen Faktoren negativ sind.

$\begin{array}{llllllllllll}0 \% & 10 \% & 20 \% & 30 \% & 40 \% & 50 \% & 60 \% & 70 \% & 80 \% & 90 \% & 100 \%\end{array}$

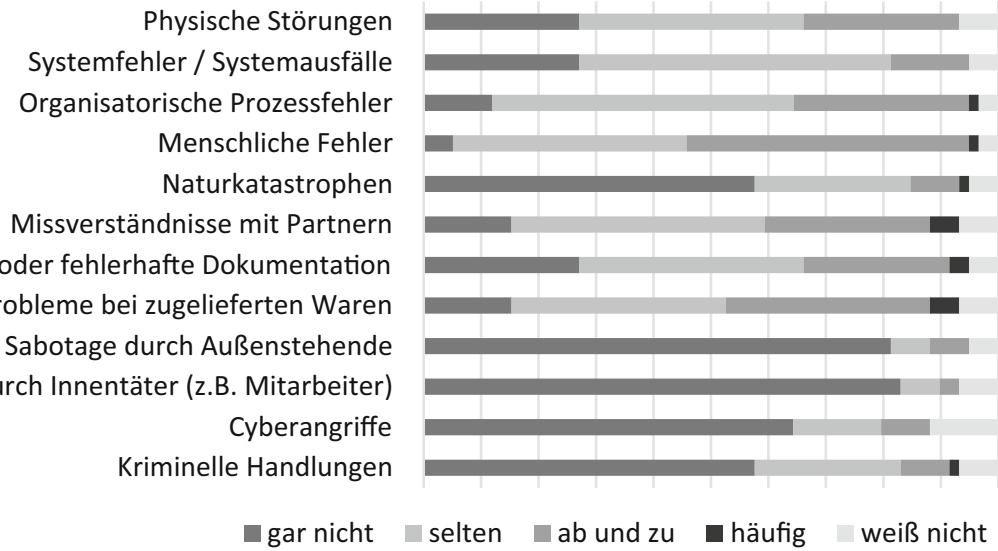

Abb. 3 Häufigkeit von Störungsursachen (NutriSafe 2020) 
Tab. 3 Korrelation des Digitalisierungsgrades mit Störungsursachen

\begin{tabular}{lll}
\hline Störungsursache & \multicolumn{2}{l}{ Einfluss des Digitalisierungsgrades } \\
& Kendall's Tau b & Signifikanz \\
\hline Systemfehler/Systemausfälle & 0,126 & 0,152 \\
Organisatorische Prozessfehler & $-0,010$ & 0,469 \\
Menschliche Fehler & $-0,138$ & 0,134 \\
Missverständnisse mit Partnern & $-0,161$ & 0,089 \\
Fehlende oder fehlerhafte Dokumentation & $-0,190$ & 0,057 \\
Cyberangriffe & 0,016 & 0,447 \\
\hline
\end{tabular}

\subsection{Verwundbarkeit}

Der Verwundbarkeit von Unternehmen drückt sich aus durch Eintrittswahrscheinlichkeiten von Unterbrechungen oder Störungen, durch die Anfälligkeit gegenüber diesen Unterbrechungen oder Störungen, durch Abhängigkeiten des Unternehmens (etwa von Infrastrukturen, Informationstechnologien, spezialisiertem Personal oder der Umwelt) sowie durch die Anpassungsfähigkeit bei Veränderungen (Abb. 4).

Die Anfälligkeit gegenüber Störungen in der Lieferkette und im eigenen Unternehmen sowie die dazugehörigen Eintrittswahrscheinlichkeiten werden von den meisten Befragten als gering bzw. sehr gering eingeschätzt. Die Einschätzung der Abhängigkeiten von Infrastrukturen, Informationstechnologien und spezialisiertem Personal fallen dagegen höher aus. Die Anpassungsfähigkeit bei Veränderungen wird überwiegend als hoch bzw. sehr hoch eingeschätzt.

In der bivariaten Analyse zwischen Digitalisierungsgrad und den Verwundbarkeitsindikatoren (Tab. 4) besteht nur bei wenigen Indikatoren ein nennenswerter Zusammenhang: Ein hoch signifikanter positiver Zusammenhang besteht zwischen

Wahrscheinlichkeit einer Störung in der Lieferkette

Anfälligkeit gegenüber Störungen in der Lieferkette

Wahrscheinlichkeit einer Störung im eigenen Unternehmen

Anfälligkeit gegenüber Störungen im eigenen Unternehmen

Abhängigkeit von Infrastrukturen (z.B. Straßen)

Abhängigkeit von Informationstechnologien

Abhängigkeit von spezialisiertem Personal

Abhängigkeit von der Umwelt (z.B. Wetter)

Anpassungsfähigkeit bei Veränderungen

sehr gering

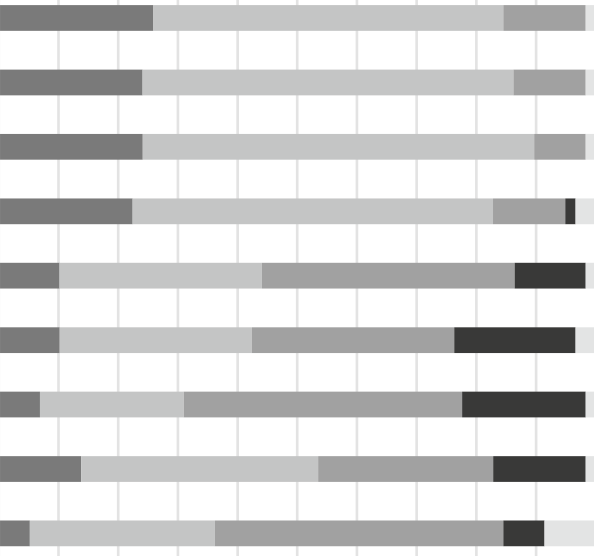

Abb. 4 Einschätzung der Verwundbarkeitsindikatoren (NutriSafe 2020) 
Tab. 4 Einfluss des Digitalisierungsgrades auf die Einschätzung der Verwundbarkeit

\begin{tabular}{lll}
\hline Verwundbarkeitsindikator (Einschätzung) & \multicolumn{2}{l}{ Einfluss des Digitalisierungsgrades } \\
& Kendall's Tau b & Signifikanz \\
\hline Wahrscheinlichkeit einer Störung in der Lieferkette & 0,068 & 0,293 \\
Anfälligkeit gegenüber Störungen in der Lieferkette & 0,084 & 0,253 \\
Wahrscheinlichkeit einer Störung im Unternehmen & 0,002 & 0,495 \\
Anfälligkeit gegenüber Störungen im Unternehmen & $-0,013$ & 0,460 \\
Abhängigkeit von Infrastrukturen & 0,076 & 0,269 \\
Abhängigkeit von Informationstechnologien & 0,326 & 0,004 \\
Abhängigkeit von spezialisiertem Personal & 0,114 & 0,173 \\
Abhängigkeit von der Umwelt & 0,048 & 0,345 \\
Anpassungsfähigkeit bei Veränderungen & 0,198 & 0,064 \\
\hline
\end{tabular}

dem Digitalisierungsgrad und der Abhängigkeit von Informationstechnologien und ein knapp nicht signifikanter positiver Einfluss des Digitalisierungsgrads besteht auf die Anpassungsfähigkeit bei Veränderungen.

Die Einschätzung der Wahrscheinlichkeit einer Störung im eigenen Unternehmen korreliert signifikant positiv mit der Häufigkeit von Systemfehlern und -ausfällen (Kendall's Tau $b=0,204$, Signifikanz $=0,046$ ). Obwohl die Kausalitätsrichtung unklar ist, kann man sinnvoll interpretieren, dass die Häufigkeit einer Störung in der Vergangenheit einen Einfluss auf die Einschätzung der Wahrscheinlichkeit für die Zukunft hat. Die Einschätzung ist damit also konsistent.

\subsection{Bewältigungskapazitäten}

Die überwiegende Mehrheit der Umfrageteilnehmer sieht sich auf die Bewältigung von Störungen im Hinblick auf die Aufrechterhaltung bzw. schnelle Wiederherstellung der Funktionsfähigkeit des Unternehmens gut vorbereitet (31\% trifft zu, 51\% trifft eher zu) und verfügt zudem nach eigener Beurteilung über die notwendigen Informationen zur Störungsbewältigung (32,8\% trifft zu, 48,3\% trifft eher zu). Allerdings gibt über die Hälfte der Befragten an, dass das Beheben einer Störung viele Ressourcen benötigt (12,1\% trifft zu, 41,4\% trifft eher zu). In der Einschätzung der Bewältigungskapazitäten gibt es keine nennenswerten Unterschiede zwischen KMU und größeren Unternehmen.

Zwischen dem Digitalisierungsgrad und den Bewältigungskapazitäten besteht kein signifikanter Zusammenhang (Tab. 5). Nur bei der Einschätzung der Vorbereitung auf die Störungsbewältigung besteht ein schwacher Zusammenhang, bei dem der Signifikanzwert nicht weit vom Signifikanzbereich entfernt ist. Unternehmen mit hohem Digitalisierungsgrad fühlen sich also besser vorbereitet auf die Störungsbewältigung.

Betrachtet man einen Zusammenhang dieser Einschätzung der Vorbereitung auf Störungsbewältigung mit anderen ausgewählten Faktoren der Resilienz (Tab. 6), gibt es drei signifikante Zusammenhänge. So besteht ein negativer Zusammenhang zwischen dem Digitalisierungsgrad und der Häufigkeit physischer Störungen (wie etwa mechanischer Defekte oder blockierter Zufahrten). Je besser sich also ein Unterneh- 
Tab. 5 Einfluss des Digitalisierungsgrades auf Bewältigungskapazitäten

\begin{tabular}{llc}
\hline Bewältigungskapazitäten & \multicolumn{1}{c}{$\begin{array}{l}\text { Einfluss des Digitalisie- } \\
\text { rungsgrades } \\
\text { Kendall's } \\
\text { Tau b }\end{array}$} & Signifikanz \\
\hline $\begin{array}{l}\text { Ich bin vorbereitet auf die Bewältigung von Störungen im Hinblick } \\
\text { auf die Aufrechterhaltung bzw. die schnelle Wiederherstellung der }\end{array}$ & 0,160 & 0,098 \\
$\begin{array}{l}\text { Funktionsfähigkeit meines Unternehmens } \\
\text { Das Beheben einer Störung in meinem Unternehmen benötigt viele } \\
\text { Ressourcen }\end{array}$ & 0,045 & 0,360 \\
$\begin{array}{l}\text { Im Falle einer Störung hat mein Unternehmen alle Informationen, die } \\
\text { zum Beheben der Störung benötigt werden }\end{array}$ & 0,156 & 0,102 \\
\hline
\end{tabular}

Tab. 6 Einfluss der Einschätzung der Vorbereitung für die Störungsbewältigung

\begin{tabular}{|c|c|c|}
\hline & \multicolumn{2}{|c|}{$\begin{array}{l}\text { Einfluss der Einschätzung der Vorbereitung für } \\
\text { Störungsbewältigung }\end{array}$} \\
\hline & Kendall's Tau b & Signifikanz \\
\hline $\begin{array}{l}\text { Häufigkeit physischer Störungen (z.B. mechani- } \\
\text { sche Defekte, blockierte Zufahrten) }\end{array}$ & $-0,215$ & 0,035 \\
\hline $\begin{array}{l}\text { Häufigkeit von Systemfehlern oder Systemausfäl- } \\
\text { len }\end{array}$ & $-0,100$ & 0,204 \\
\hline $\begin{array}{l}\text { Einschätzung der Anfälligkeit gegenüber Störun- } \\
\text { gen in der Lieferkette }\end{array}$ & 0,329 & 0,001 \\
\hline $\begin{array}{l}\text { Einschätzung der Anfälligkeit gegenüber Störun- } \\
\text { gen im eigenen Unternehmen }\end{array}$ & 0,221 & 0,021 \\
\hline
\end{tabular}

men auf die Störungsbewältigung vorbereitet einschätzt, desto seltener sind physische Störungen. Ein positiver Zusammenhang dagegen besteht bei der Einschätzung der Anfälligkeit gegenüber Störungen in der Lieferkette (höchst signifikant) sowie im eigenen Unternehmen. Das könnte auf Sensibilisierungseffekte durch die Auseinandersetzung mit beispielsweise Risiken im Rahmen der Vorbereitung auf die Störungsbewältigung zurückzuführen sein.

\section{Limitationen und Fazit}

Da die Umfrageteilnehmer zu großen Teilen digital angesprochen wurden und ein digitaler Themenbezug kommuniziert wurde, können die Ergebnisse der Studie durch Selbstselektion zugunsten der Digitalisierung verzerrt sein. Auch ist die Stichprobengröße für eine Übertragung auf die Grundgesamtheit nicht ausreichend. Die Ergebnisse können also nicht ohne weiteres auf die Grundgesamtheit übertragen werden, sondern gelten in erster Linie für das Sample. Insgesamt sind die Ergebnisse aufschlussreich, um den Themenkomplex der Digitalisierung und Resilienz in der Lebensmittelproduktion und -logistik zu beleuchten.

Auffällig ist, dass die meisten Betriebe mindestens digital unterstützt sind und dass die häufigsten Störungsursachen menschlicher Natur sind und weniger auf technische Ursachen zurückgehen. Aber auch diese Störungen kommen vor. Bei der 
Einschätzung der Verwundbarkeit werden Eintrittswahrscheinlichkeiten und Impact von Störungen als gering eingeschätzt, während Abhängigkeiten höher eingeschätzt werden. Die Abhängigkeit von Informationstechnologien spielt dabei besonders bei digitalisierten Betrieben eine Rolle.

Der Digitalisierungsgrad hat einen Einfluss auf Faktoren der Resilienz. So sind Zusammenhänge zwischen dem Digitalisierungsgrad und technischen Störungsursachen positiv und zwischen dem Digitalisierungsgrad und menschlichen bzw. organisatorischen Störungsursachen negativ. Es scheint also, dass die Digitalisierung auf der einen Seite zu mehr technischen Störungen führt und auf der anderen Seite zu einer Reduktion an menschlichen und organisatorischen Fehlern. Weitere Zusammenhänge bestehen zwischen dem Digitalisierungsgrad und bestimmten Verwundbarkeitsindikatoren. So besteht ein Zusammenhang zwischen dem Digitalisierungsgrad und der Abhängigkeit von Informationstechnologien und die Anpassungsfähigkeit bei Veränderungen profitiert von der Digitalisierung.

Bisher ist wenig über das Thema Resilienz in kleinen und mittleren Unternehmen der Lebensmittelproduktion und -logistik bekannt. Der vorliegende Beitrag fügt sich in diese Lücke und gibt ein Lagebild der Digitalisierung und Resilienz in diesen Unternehmen sowie der Zusammenhänge zwischen Digitalisierung und Resilienz. Besonders im Kontext voranschreitender Digitalisierung ist es hilfreich, mögliche Auswirkungen auf die Lebensmittelversorgung zu kennen. So birgt die Digitalisierung von Prozessen auf der einen Seite Chancen zur Minderung menschlicher Fehler und erhöht stellenweise auch die Kapazitäten zur Bewältigung von Störungen, andererseits ist das auch mit Risiken vor allem in Bezug auf einen Anstieg technischer Störungen oder zusätzlichen Abhängigkeiten verbunden.

Danksagung Wir danken dem Bundesministerium für Bildung und Forschung (BMBF) für die Möglichkeit der Forschung im Rahmen des Projektes NutriSafe (FKZ 13N15070 bis 13N15076) sowie dem Sicherheitsforschungsförderprogramm KIRAS, finanziert vom Bundesministerium für Landwirtschaft, Regionen und Tourismus (Projektnummer: 867015). Ferner danken wir den Umfrageteilnehmern für die Zeit und den Multiplikatoren, die uns bei der Streuung der Umfrage unterstützt haben.

Funding Open Access funding provided by Projekt DEAL.

Open Access Dieser Artikel wird unter der Creative Commons Namensnennung 4.0 International Lizenz veröffentlicht, welche die Nutzung, Vervielfältigung, Bearbeitung, Verbreitung und Wiedergabe in jeglichem Medium und Format erlaubt, sofern Sie den/die ursprünglichen Autor(en) und die Quelle ordnungsgemäß nennen, einen Link zur Creative Commons Lizenz beifügen und angeben, ob Änderungen vorgenommen wurden.

Die in diesem Artikel enthaltenen Bilder und sonstiges Drittmaterial unterliegen ebenfalls der genannten Creative Commons Lizenz, sofern sich aus der Abbildungslegende nichts anderes ergibt. Sofern das betreffende Material nicht unter der genannten Creative Commons Lizenz steht und die betreffende Handlung nicht nach gesetzlichen Vorschriften erlaubt ist, ist für die oben aufgeführten Weiterverwendungen des Materials die Einwilligung des jeweiligen Rechteinhabers einzuholen.

Weitere Details zur Lizenz entnehmen Sie bitte der Lizenzinformation auf http://creativecommons.org/ licenses/by/4.0/deed.de. 


\section{Literatur}

Bundesamt für Bevölkerungsschutz und Katastrophenhilfe (2011) Glossar - R. https://www.bbk.bund.de/ SubSites/Kritis/DE/Servicefunktionen/Glossar/Functions/glossar.html?lv2=4968608. Zugegriffen: 2. Juni 2020

Bundesamt für Sicherheit in der Informationstechnik (o.J.) Glossar der Cybersicherheit. https://www.bsi. bund.de/DE/Themen/Cyber-Sicherheit/Empfehlungen/cyberglossar/Functions/glossar.html?cms_ lv2=9817306. Zugegriffen: 2. Juni 2020

Bundesamt für Sicherheit in der Informationstechnik (2019) Die Lage der IT-Sicherheit in Deutschland 2019

Bundesministerium des Innern (2016) Verordnung zur Bestimmung Kritischer Infrastrukturen nach dem BSI-Gesetz (BSI-KritisV)

Christopher M, Peck H (2004) Building the resilient supply chain. Int J Logist Manag 15(2):1-14

Deutscher Bundestag (2015) Gesetz zur Erhöhung der Sicherheit informationstechnischer Systeme (ITSicherheitsgesetz). Bundesgesetzblatt (Jahrgang 2015, Teil I, Nr. 31)

Europäische Kommision (2019) RASFF annual report 2018. Publications Office of the European Union, Luxemburg

Europäische Kommission (2003) Empfehlung der Kommission vom 6. Mai 2003 betreffend der Definition der Kleinstunternehmen sowie der kleinen und mittleren Unternehmen. AB1. L 124 vom 20. Mai 2003

Eurostat (2008) NACE Rev. 2 - Statistische Systematik der Wirtschaftszweige in der Europäischen Gemeinschaft. Amt für amtliche Veröffentlichungen der Europäischen Gemeinschaften, Luxemburg.

Nutrisafe (2019) NutriSafe - Sicherheit in der Lebensmittelproduktion und -logistik durch die DistributedLedger-Technologie. https://nutrisafe.de. Zugegriffen: 2. Juni 2020

NutriSafe (2020) NutriSafe Monitor - Resilienz und Blockchain-Technologie in Lebensmittelproduktion und -logistik. https://nutrisafe.de/monitor. Zugegriffen: 2. Juni 2020

Platz U (2005) Vulnerabilität von Logistikstrukturen im Lebensmittelhandel. Landwirtschaftsverlag, Münster-Hiltrup.

Ponis ST, Koronis E (2012) Supply chain resilience - definition of concept and its formative elements. J Appl Bus Res 28(5):921 (Clute Institute)

Reimers T, Rudel S, Lechner U, Hofmeier M, Wilhelmi T, Fikar C, Kipker D, Buchner B, Sohr K, Kus M, Blunk R, Schuster M, Bensch P, Bollen T, Krummel V, Le DK, Friedrich H, Joseph S, Furtner T, Hirsch P, Leithner M, Semmelmann K, Weber C, Müller S, Brändle J, Domig K, Göllner J, Laßnig H, Springnagel M, Zazgornik J (2019) Absicherung von Wertschöpfungsketten in der Lebensmittelproduktion und -logistik mittels Distributed-Ledger-Technologie - Das Forschungsdesign. In: Bundesamt für Sicherheit in der Informationstechnik (Hrsg) Tagungsband zum 16. Deutschen ITSicherheitskongress, Bonn

VeSiKi (2018) Monitor 2.0 IT-Sicherheit Kritische Infrastrukturen. https://monitor.itskritis.de. Zugegriffen: 2. Juni 2020 